\title{
Effect of electron disruption in the energy recovery linac based electron ion collider
}

\author{
Y. Hao and V. Ptitsyn \\ Brookhaven National Laboratory, Upton, New York 11973, USA
}

(Received 2 February 2010; published 19 July 2010)

\begin{abstract}
Beam-beam effects present one of the major factors limiting the luminosity of colliders. In the energy recovery linac (ERL) based eRHIC design, the electron beam, accelerated in a superconducting ERL, collides with the proton beam circulating in the RHIC ring. During such collisions the electron beam undergoes a very strong beam-beam interaction with the protons, which warrants careful examination. We evaluated transverse disruption and linear mismatch effects in the electron beam caused by collisions and considered several countermeasures to mitigate the emittance growth from these interactions. The minimum required aperture of transport lines is calculated that should allow the transport of the electron beam during the deceleration process.
\end{abstract}

DOI: 10.1103/PhysRevSTAB.13.071003

PACS numbers: 29.27.Bd, 29.20.db

\section{INTRODUCTION}

The design of eRHIC, based on an energy recovery linac (ERL), opens a way to luminosities exceeding $10^{33} \mathrm{~cm}^{-2} \mathrm{~s}^{-1}[1,2]$. In this configuration, the electron accelerator includes a superconducting energy recovery linac and four recirculating passes that accelerate the electron beam to $10 \mathrm{GeV}$, with the possibility of an extension to higher energies. The electron beam collides with the proton beam circulating in one of the RHIC rings, after which the electron beam must be decelerated in the same linac, so that the energy, taken away from the electron bunches in the linac cavities, can be reused in accelerating other bunches. Table I lists the basic parameters of the present eRHIC linac-ring design; the values in parentheses correspond to the suggested modification after parameter optimization described in this paper.

In eRHIC, the strength of the beam-beam force acting on the electron beam is much larger than that acting on the proton beam, taking full advantage of the fact that the electron beam is used only once for the collisions. The beam-beam parameters of electrons and protons, listed in Table I, differ by 2 orders of magnitude. The electron beam is disrupted considerably by the beam-beam force just after one collision with the opposing proton beam, while the proton beam's distribution changes very slowly. In investigating the evolution of the electron beam in one collision process, we reasonably can assume that the proton beam is rigid. Two different components are distinguishable in the disruption effects of the electron beam. First, the nonlinearity of the beam-beam force distorts its distribution at high transverse amplitudes and increases its rms emittance. Second, the linear part of the beam-beam interaction (strong focusing force) causes a mismatch in the electron distribution in the phase space compared with the aperture shape, as defined by the design lattice in the absence of collisions. Both effects must be considered to select optimal beam and lattice parameters and to evaluate the mini- mal aperture of the transport line elements needed to transport the beam during its deceleration on the energy recovery path.

\section{THE BEAM-BEAM INTERACTION MODEL AND SIMULATION CODE}

For a transverse Gaussian distribution beam with rms size $\sigma_{x}$ and $\sigma_{y}$, the electric field generated by it can be represented by the Bassetti-Erskine formula [3]. In the ERL-based eRHIC, both beams are round at the collision point. The electric field has a very simple form:

$$
\vec{E}_{r}=\frac{n(z) e}{2 \pi \varepsilon_{0} r^{2}}\left[1-\exp \left(-\frac{r^{2}}{2 \sigma^{2}}\right)\right] \vec{r},
$$

where $n(z)$ is the longitudinal charge density and $\sigma$ is the beam's transverse beam size. The Lorentz force gives the following form:

$$
\vec{F}=e\left(\vec{E}_{r}+\vec{v} \times \vec{B}_{\theta}\right)=2 e \vec{E}_{r}
$$

The latter simplification is only valid for eRHIC where the beam velocities are very close to speed of light and no crossing angle is included.

We assume that at the interaction region there are no external electromagnetic fields, so that the only force exerted on the electron beam is the beam-beam force from the proton beam. Near the axis, this force is linear. The motion of a single electron can be calculated from Hill's equation:

$$
x^{\prime \prime}+k^{2}(s) x=0 .
$$

Here, we use $s$ as longitudinal coordinate and $k(s)$ represents the beam-beam interaction from the opposing beam. We set the interaction point (IP) at $s=0$, and a positive $s$ corresponds to the head of the proton bunch. Consequently, the electron beam travels from a positive $s$ to a negative one, and oscillates inside the proton beam because the beam-beam interaction is an attractive force in both transverse directions. Here, we ignore the longitudinal structure 
TABLE I. ERL-based eRHIC beam parameters.

\begin{tabular}{lcccc}
\hline \hline & \multicolumn{2}{c}{ High-energy setup } & \multicolumn{2}{c}{ Low-energy setup } \\
& $p$ & $e$ & $p$ & $e$ \\
\hline Energy $(\mathrm{GeV})$ & 250 & 10 & 50 & 3 \\
Number of bunches & 166 & & 166 & \\
Bunch intensity $\left(10^{11}\right)$ & 2.0 & 1.2 & 2.0 & 1.2 \\
Beam current $(\mathrm{mA})$ & 420 & 260 & 420 & 260 \\
$95 \%$ normalized emittance $(\pi \mathrm{mm}$ mrad) & 6 & 115 & 6 & 115 \\
rms emittance $(\mathrm{nm}$ rad) & 3.8 & $1.0 /(5.0)$ & 19.0 & $3.3 /(16.5)$ \\
$\beta^{*}(\mathrm{~cm})$ & 26 & $100 /(20)$ & 26 & $150 /(30)$ \\
Beam-beam parameter & 0.015 & $2.3 /(0.46)$ & 0.015 & $2.3(0.46)$ \\
Disruption parameter & 0.005 & 5.8 & 0.01 & 3.8 \\
rms bunch length $(\mathrm{cm})$ & 20 & 0.7 & 20 & 1.5 \\
Polarization $(\%)$ & 70 & 80 & 70 & 80 \\
Peak luminosity $\left(10^{33} \mathrm{~cm}^{-2} \mathrm{~s}^{-1}\right)$ & \multicolumn{2}{c}{0.53} \\
\hline \hline
\end{tabular}

of electron beam because its bunch length is about 30 times shorter than the proton ones. If $\lambda_{s}(s)$ is denoted as the longitudinal proton beam density that thin electron bunch meets at $s$, we have $\int \lambda_{s}(s) d s=1$, and $k(s)$ has the following form:

$$
k^{2}(s)=\frac{N_{p} r_{e}}{\sigma_{p x}^{2} \gamma_{e}} \lambda(s)=\frac{1}{f_{e}} \lambda_{s}(s),
$$

where $\sigma_{p x}$ is transverse rms size of the proton beam that remains constant provided that the hourglass effect is excluded; $r_{e}$ is the electron's classical radius and $f_{e}$ is the focal length of beam-beam field of the electron beam. In simulations or in a numerical solver of Hill's equation, the hourglass effect is easily implemented by varying $\sigma_{p x}$. Denoting $z$ as the longitudinal deviation of the proton beam slice with respect to the reference particle, the longitudinal position where the electron bunch collides with a thin proton beam slice at $z$ always is located at $s=z / 2$, because the electron bunch is assumed infinite thin. Therefore, we can rewrite $\lambda_{s}(s)$ from the proton's real longitudinal distribution $\lambda_{z}(z)$. The relation between two functions yields

$\int_{-\infty}^{\infty} \lambda_{s}(s) d s=\int_{-\infty}^{\infty} \lambda_{z}(z) d z \stackrel{z=2 s}{\rightarrow} 2 \int_{-\infty}^{\infty} \lambda_{z}(2 s) d s$

$\lambda_{s}(s=z / 2)=2 \lambda_{z}(2 s)$.

After establishing this relationship, we later use $\lambda(s)=$ $\lambda_{z}(s)$ for simplicity. Usually, Gaussian distribution is a good model for longitudinal position, viz.,

$$
\lambda(z)=\lambda_{z}(z)=\frac{1}{\sqrt{2 \pi} \sigma_{p z}} \exp \left(-\frac{z^{2}}{2 \sigma_{p z}^{2}}\right) .
$$

Here, $\sigma_{p z}$ is the rms bunch length of the proton beam.

The disruption parameter, $d$, is defined as

$$
d_{e}=\sigma_{p z} / f_{e}=\frac{N r_{e} \sigma_{p z}}{\sigma_{p x}^{2} \gamma_{e}} .
$$

The definition gives 5.8 for the electron beam using eRHIC's parameters. Now Eq. (4) and Hill's equation can be expressed with the disruption parameter as

$$
\begin{gathered}
k^{2}(s)=\frac{N_{p} r_{e}}{\sigma_{p x}^{2} \gamma_{e}} \lambda_{s}(s)=\frac{2 d_{e}}{\sigma_{p z}} \lambda(2 s) \\
x^{\prime \prime}+\frac{2 d_{e}}{\sigma_{p z}} \lambda(2 s) x=0 .
\end{gathered}
$$

The wave number of electron oscillation can be calculated from Eq. (8), following the procedure in [4]:

$$
\begin{aligned}
n & =\frac{1}{2 \pi} \int_{-\infty}^{\infty} k(s) d s=\frac{1}{2 \pi} \sqrt{\frac{2 d_{e}}{\sigma_{p z}}} \int_{-\infty}^{\infty} \sqrt{\lambda(2 s)} d s \\
& =\frac{\sqrt{d_{e}}}{(2 \pi)^{3 / 4}} \approx \frac{\sqrt{d_{e}}}{4} .
\end{aligned}
$$

Thus, a single electron near the beam's axis will finish $0.25 \times \sqrt{5.78}=0.6$ periods of oscillation. In the same time, the envelope of the electron beam will make $2 \times$ $0.6=1.2$ periods of oscillation.

To study the beam-beam interaction with a full nonlinear force, we can employ a strong-weak simulation code because the proton beam is nearly unchanged during single collision. To simulate the beam-beam effects for ERLbased eRHIC, we wrote a dedicated code [5], named EPIC. The EPIC code supports comprehensive explorations of the various features of the beam-beam interaction for a linac-ring collision scheme. For studying the electron beam's disruption, we can fix the parameters of the proton beam in the code, providing the strong-weak simulation mode. The longitudinal Gaussian distribution of the proton beam has a cutoff at $\pm n \sigma_{p z}$, where $n$ is typically selected as 4 . The proton bunch is cut into multiple slices; for the eRHIC's parameters, 20 or more slices lead to consistent results. Each slice is treated as an infinitely short bunch with a transverse Gaussian distribution generating the electric field according to Eq. (1). The slicing procedure 
accounts for the variation of the transverse rms beam size of the proton beam due to the hourglass effect (the proton beta-function variation).

The short electron beam is represented by macroparticles that experience consecutive kicks from the beambeam interactions with proton slices. The macroparticles can be generated with a desirable initial transverse distribution, for instance, a Gaussian or Beer-Can (uniform 2D transverse distribution with a hard edge at $2 \sigma$ ) type. Usually the electron beam produced at source is assumed to be a Beer-Can distribution. It takes damping time (usually milliseconds) for the distribution to change to Gaussian type because of the radiation damping and excitation. The electron beam emittance, beam size, details of the distribution, and the luminosity can be calculated by postprocessing the macroparticle data. Usually, 50 thousand macroparticles suffices to determine beam emittance, optics functions, and other statistics quantities. However, for studying power loss, the number of macroparticles is decided upon the accuracy because each one represents a significant amount of beam energy.

\section{THE MISMATCH EFFECT}

Without the beam-beam effect, the electron beam's transverse distribution matches the phase space ellipse defined by the optical functions (alpha and beta functions) of the design lattice. The focusing effect of the beam-beam force distorts the optical functions, so that the phase space distribution of the electron beam is incongruous with the design lattice. Since the electron beam after collision must be transported and decelerated, it is important to minimize the mismatch caused by beam-beam interactions.

Quantifying the phase space mismatch entails comparing the beta and alpha functions calculated with and without the beam-beam interaction. Both functions can be obtained from the envelope equation [6], expressed as

$$
\begin{gathered}
w^{\prime \prime}+k^{2}(s) w-1 / w^{3}=0 \quad \beta(s)=w^{2}(s) \\
\alpha(s)=-w(s) w^{\prime}(s) .
\end{gathered}
$$

The solutions of this equation in the drift space $[k(s)=0]$ are well known:

$$
\begin{aligned}
& \beta(s)=\frac{1}{\gamma(0)}+\gamma(0)\left(s-\frac{\alpha(0)}{\gamma(0)}\right)^{2} \\
& \alpha(s)=\alpha(0)-\gamma(0) s .
\end{aligned}
$$

The solutions with the beam-beam force, with $k(s)$ defined by Eq. (4), should be obtained numerically in the general case by a differential equation solver. Theoretically, the boundary condition is at negative infinity away from the IP before collision. Because of the rapid attenuation of the proton beam's longitudinal distribution beyond $4 \mathrm{rms}$ bunch length, we can set the boundary condition at the entrance of interaction region (IR) drift where $s=3 \mathrm{~m}$. By solving the differential equation with nonzero $k(s)$, we obtain the evolution of the beta and alpha function in the whole IR drift with beam-beam interaction. Then the two cases, with or without beam-beam effect, can be easily compared.

If the waist beta function $\left(\beta^{*}\right)$ is $1 \mathrm{~m}$ and the waist position $\left(s^{*}\right)$ is at IP $\left(s^{*}=0\right)$, the solution of beta and alpha function in IR is that shown in Figs. 1(a) and 1(b).

In Fig. 1, for a large positive longitudinal position, the two curves overlap with each other because the beam-beam force is too weak to separate them. The mismatch becomes huge near and after the proton bunch's center if $\beta^{*}$ is $1 \mathrm{~m}$. The numerical results of $\beta^{*}=0.2 \mathrm{~m}$ are shown in Figs. 1(c) and 1(d). The discrepancy between two curves is much smaller than the case $\beta^{*}=1 \mathrm{~m}$. Comparing these two cases, it is notable that, for $\beta^{*}=1 \mathrm{~m}$, the beta function after the beam-beam collision is larger than without the beam-beam effect; while for $\beta^{*}=0.2 \mathrm{~m}$, the result is opposite. We expect to have proper $\beta^{*}$ for perfect matching. Hence, we are interested in the solution of

$$
\beta\left(\beta^{*}, s=-3 m\right)=\beta^{*}+\frac{s^{2}}{\beta^{*}} .
$$

Its numerical solution yields $\beta^{*}=0.225 \mathrm{~m}$. In Fig. 2, we show the case with a perfect match. The betatron amplitude functions with the beam-beam effect exactly match those functions without the beam-beam force except longitudinal position near IP. It is impossible to distinguish whether there is collision by just measuring the electron beam at IP downstream.

The value of $\beta^{*}$ for exact matching depends on the parameters of the proton beam (intensity, transverse emittance, bunch length) and does not always exist. For a uniform longitudinal distribution of the proton beam, the exactly matched solution can be found analytically. If the proton beam has constant longitudinal profile within $[-2 L, 2 L]$, the proton rms beam size is $2 L / \sqrt{3}$, and $k(s)=$ $\left(2 f_{e} L\right)^{-1 / 2}$. Using the transfer map of thick quadrupole and drift space, we can calculate the exact matching solution as

$$
\beta^{*}=\frac{\sqrt{1-k^{2} L^{2}+k L[\tan (k L)-\cot (k L)]}}{k} .
$$

When $k L$ falls in the region $[0, \pi / 2]$, the matching solution always exists. Equivalently, this requires that the disruption parameter $D$ less than $\pi^{2} / \sqrt{3}=5.7$.

For Gaussian longitudinal distribution, the requirement for the maximum disruption parameter is obtained numerically. For the proton beam with rms bunch length of $0.2 \mathrm{~m}$, the disruption parameter, which assures a matched optics solution, should be less than 8.3 .

Although we found that for eRHIC beam parameters the electron $\beta^{*}$ of $0.225 \mathrm{~m}$ at the interaction point eliminates 

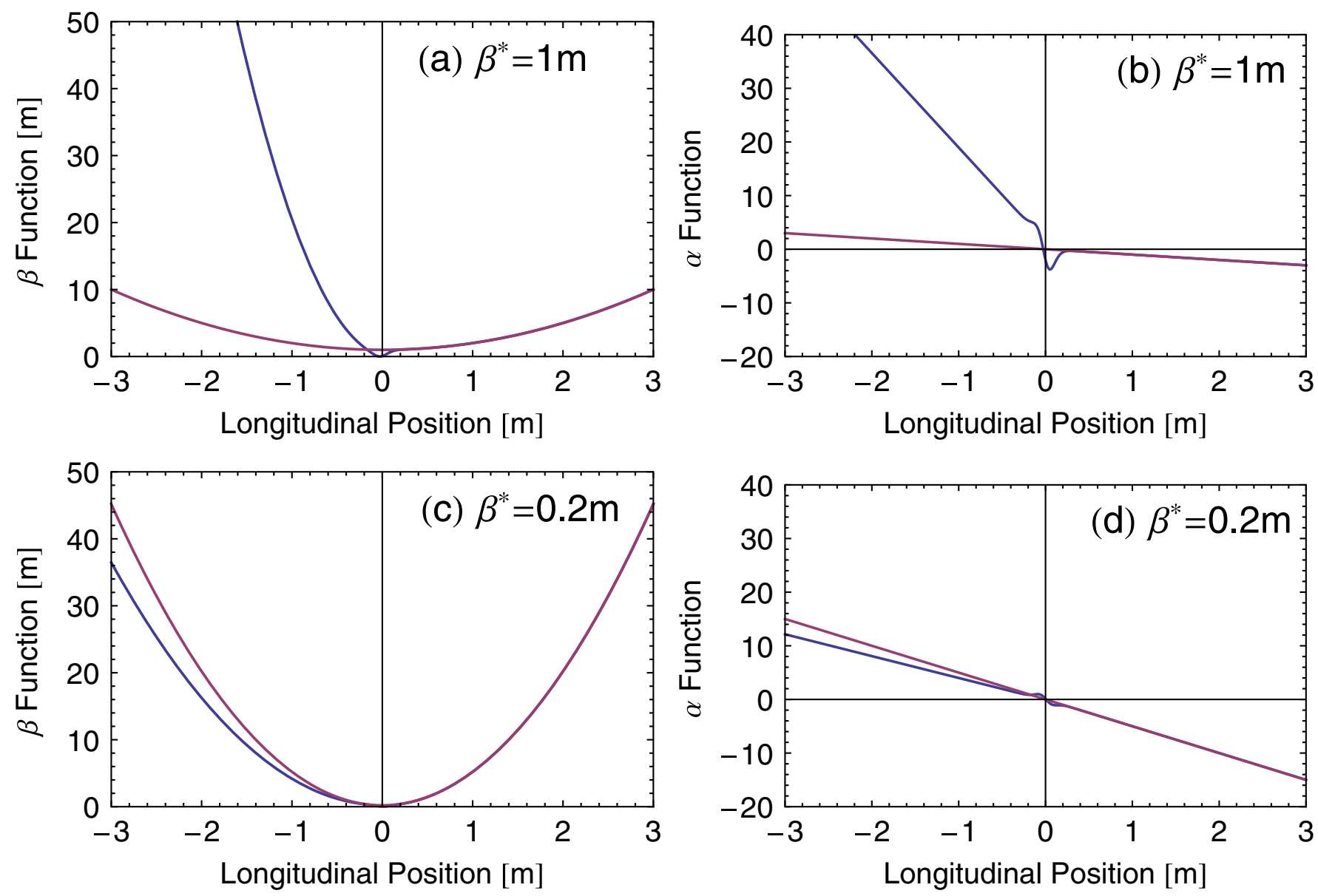

FIG. 1. (Color) The numerical solution of alpha and beta functions in IR. The solution of Eqs. (10) is the blue curve, while the red curve corresponds to the solution without beam-beam force. In (a) and (b), $\beta^{*}=1 \mathrm{~m}$; in (c) and (d) $\beta^{*}=0.2 \mathrm{~m}$. In all graphs, $s^{*}=0$.

the mismatch of linear optics after the beam-beam interaction, we consider that the nonlinear part of the beambeam force should be accounted in finally determining optimally designed optical functions.

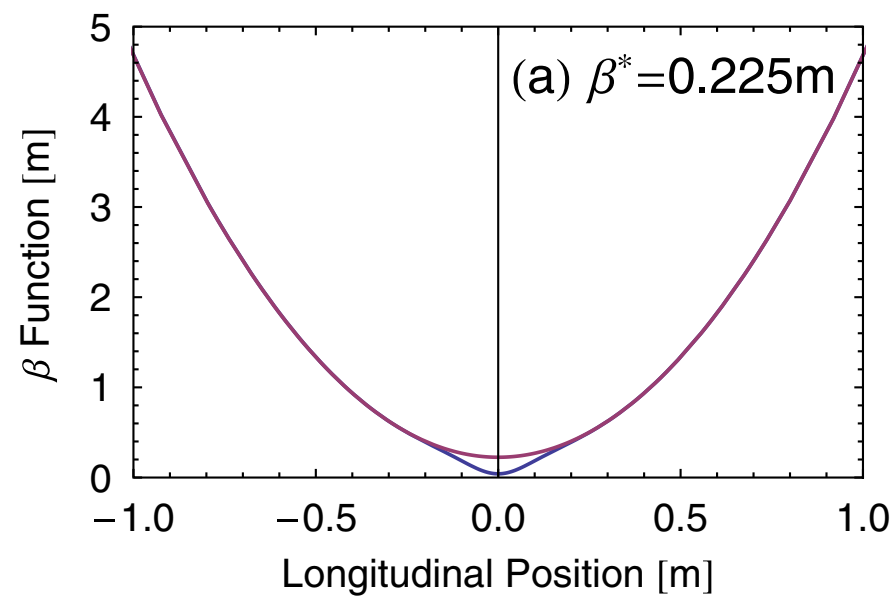

\section{NONLINEAR DISRUPTION EFFECT}

In the previous section, we discussed the electron distribution mismatch under a linear beam-beam force. Those results are obtained by solving a linear differential equa-

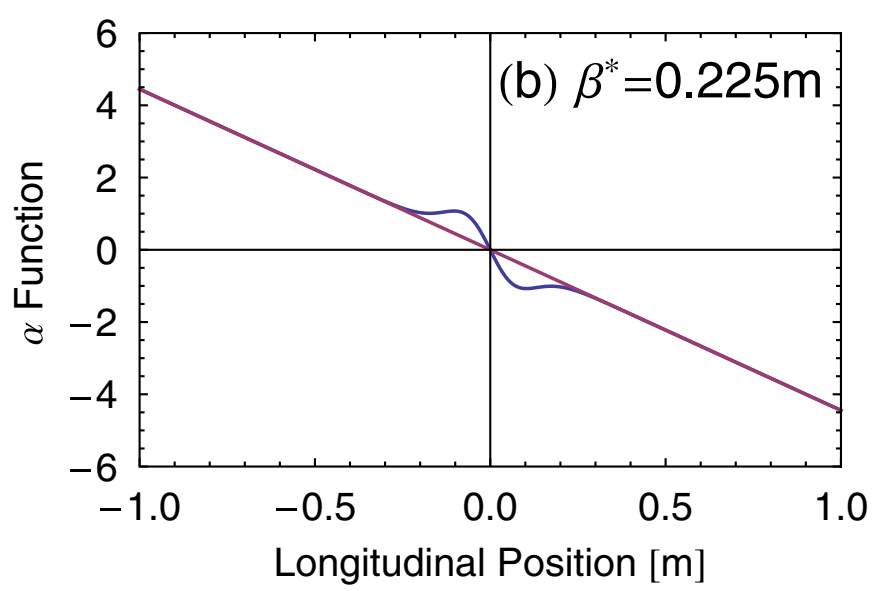

FIG. 2. (Color) The numerical solution of Eqs. (10) is the blue curve, while the red curve corresponds to betatron amplitude function without beam-beam force. In (a) and (b), the initial $\beta^{*}$ is $0.225 \mathrm{~m}$, with $s^{*}=0$. 
tion (10). However, for electrons that are far from the axis, the force of beam-beam interaction is highly nonlinear, such that it distorts the electron distribution, especially that of large betatron amplitudes. Consequently, the geometric emittance of the electron beam is altered. To explore the resulting deterioration of the beam emittance, we employ the simulation code.

First, we calculated the evolution of the electron beam size and its emittance using strong-weak simulation with the parameters in Table I. The design electron $\beta^{*}$ is $1 \mathrm{~m}$ at $s^{*}=0 \mathrm{~m}$. We consider the $z$ coordinate $(z=2 s)$ to range from -1 to $1 \mathrm{~m}$ relative to the reference particle, which corresponds to $\pm 5 \sigma \mathrm{\sigma z}$ of the proton bunch length. The actual collision occurs from -0.5 to $0.5 \mathrm{~m}$ relative to the IP. Figure 3 illustrates the evolution of rms beam size and rms emittance.

In Fig. 3, the electron beam travels from positive longitudinal position to negative position. We see that the rms electron beam emittance (red curve) increases by a factor of 2 during the entire collision process. Most of the gain happens in the range of $[-0.2 \mathrm{~m}, 0.2 \mathrm{~m}]$ which corresponds to the interaction with the proton beam within $\pm 2 \sigma_{p z}$. Because of the strong focusing of the proton beam, the electron beam is pinched, thereby having a smaller transverse size.

Under the nonlinear force, the distribution in phase space of the electron beam is warped from its original elliptic shape in the transverse phase space, as shown in Fig. 4. To easily compare this change with the design optics, unperturbed by the beam-beam effect, we transferred all macroparticle coordinates after beam-beam interaction back to the IP position. Mathematically, the process is treated as

$$
\begin{aligned}
& \tilde{x}_{e}(s=0)=x_{e}(s)-x_{e}^{\prime}(s) s \\
& \tilde{y}_{e}(s=0)=y_{e}(s)-y_{e}^{\prime}(s) s
\end{aligned}
$$

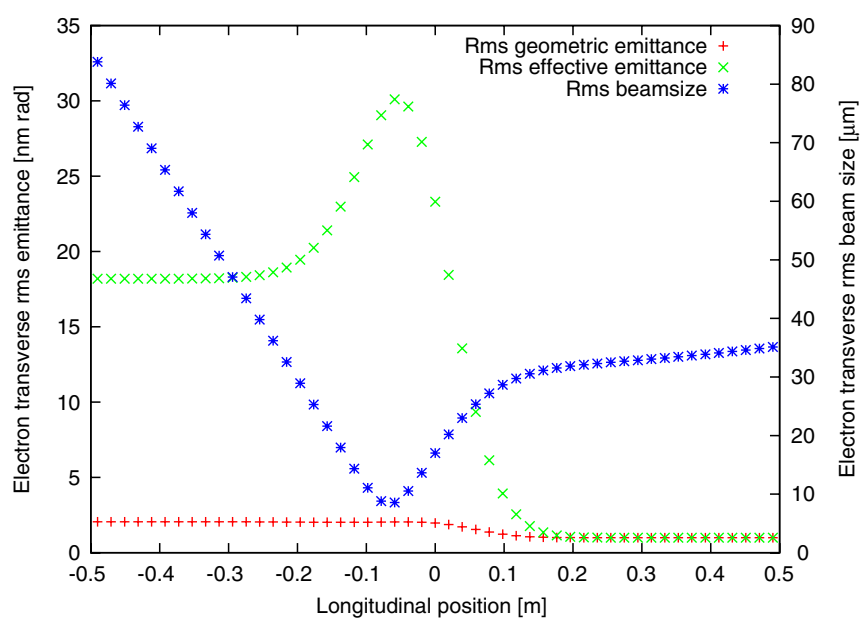

FIG. 3. (Color) Evolution of beam size and rms emittance (geometric and effective) of the electron beam for $\beta^{*}=1 \mathrm{~m}$ at $s^{*}=0 \mathrm{~m}$.

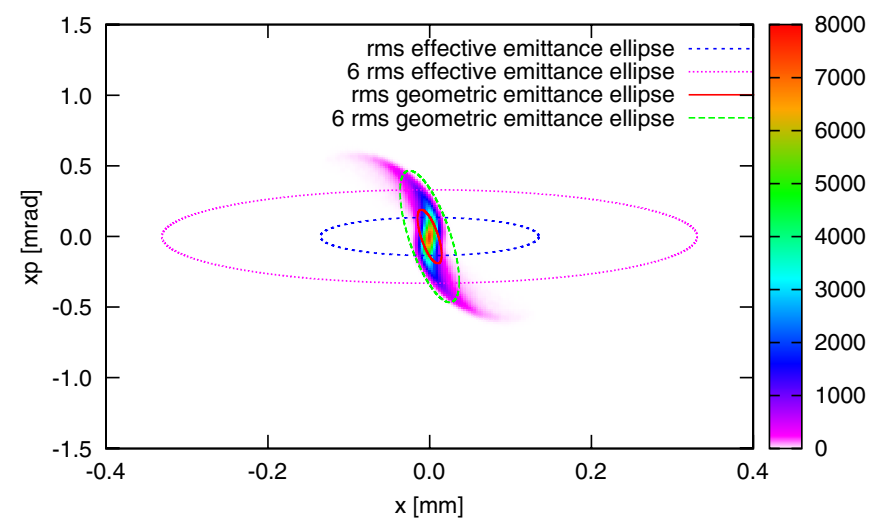

FIG. 4. (Color) Phase space distribution of the electron beam after collision for $\beta^{*}=1 \mathrm{~m}$, back traced to the IP. The rms and 6 rms ellipses for both geometric and effective emittance, respectively, are plotted.

with $s=-0.5 \mathrm{~m}$. The coordinates with tildes represent those at IP. After obtaining the virtual distribution at IP, we can calculate the modified optical functions on the basis of second order momenta of the electron beam distribution:

$$
\left(\begin{array}{cc}
\beta_{x} & -\alpha_{x} \\
-\alpha_{x} & \gamma_{x}
\end{array}\right)=\frac{1}{\varepsilon_{x}}\left(\begin{array}{cc}
\left\langle x^{2}\right\rangle & \left\langle x x^{\prime}\right\rangle \\
\left\langle x x^{\prime}\right\rangle & \left\langle x^{\prime 2}\right\rangle
\end{array}\right)
$$

Here, $\varepsilon_{x}$ represents the rms geometric emittance obtained from the beam distribution, written as

$$
\varepsilon_{x}=\sqrt{\left\langle(x-\bar{x})^{2}\right\rangle\left\langle\left(x^{\prime}-\bar{x}^{\prime}\right)^{2}\right\rangle-\left\langle(x-\bar{x})\left(x^{\prime}-\bar{x}^{\prime}\right)\right\rangle^{2}} .
$$

Figure 4 shows the modification of the transverse beam distribution due to the beam-beam interaction. Without beam-beam interaction (the design lattice), at IP the alpha function vanishes and the Courant-Snyder invariant ellipse is oriented upright with the ratio of its semiaxis equal to $1 / \beta^{*}$. The beam-beam interaction introduces, as already discussed, a large mismatch between the phase space distributions and the design lattice. However, in the presence of the nonlinear beam-beam field the beta and alpha functions, which characterize the modified distribution in the phase space, deviate from those functions calculated from the linear mismatch. From Eq. (15), we found the modified beta and alpha functions at IP:

$$
\tilde{\beta}(s=0)=0.11 \mathrm{~m} \quad \tilde{\alpha}(s=0)=0.94 .
$$

After the collision, the electron beam is decelerated in the process of energy recovery, and travels through several recirculation passes. The final electron emittance after the collision is very important, since the magnets of recirculation passes as well as components of the superconducting linac must have a sufficiently large aperture to accommodate the disrupted electron beam and avoid beam losses. To determine the acceptable magnet apertures, it is reasonable to consider that the final electron beam emittance is not defined as the geometric emittance, but rather, according to 
the emittance shape from the design lattice without collisions, diluting the real beam distribution in betatron phase to fill the emittance shape defined by the design lattice. Mathematically, the geometric emittance is defined in Eq. (16) and the effective emittance is defined as the half of the average value of Courant-Snyder invariant of all macroparticles based on design lattice:

$$
C\left(\tilde{x}, \tilde{x}^{\prime}\right)=\gamma \tilde{x}^{2}+2 \alpha \tilde{x} \tilde{x}^{\prime}+\beta \tilde{x}^{\prime 2} .
$$

For the example of $\beta^{*}=1 \mathrm{~m}$, we compare, in Fig. 3, the evolution of rms geometric emittance and effective emittance. We note that the increase of the rms effective emittance due to the mismatch is much larger than that of geometric emittance related to the nonlinear beam-beam force. Before the collision, the geometric emittance and the effective emittance have the same value because the beam distribution perfectly matches the optics. After the collision, in this case, the final rms effective emittance is about 9 times larger than geometric rms emittance, and 18 times larger than original rms value. However, the increases in both geometric and effective rms emittances depend on the choice of the design optics.

From Sec. II, we know that the lattice mismatch mainly is a linear effect. Expectedly, the mismatch can be reduced by properly choosing the betatron amplitude functions of the electron beam, as calculated in Sec. II. Since a smaller design $\beta^{*}$ reduces the mismatch, we can plot the rms emittance growth and rms beam size evolution throughout the interaction region, for $\beta^{*}=0.2 \mathrm{~m}$. To maintain the same size of the electron beam at IP, the initial emittance value must be correspondingly increased to $5 \mathrm{~nm} \mathrm{rad}$.

From Fig. 5, we can observe two advantages over large design $\beta^{*}=1 \mathrm{~m}$. First the growth of rms geometric emittance due to the collision is low for low $\beta^{*}$; it is only about $4 \%$ in contrast to $100 \%$ for $\beta^{*}=1 \mathrm{~m}$. Second, the rms effective emittance after interaction has about a $25 \%$ increment, far less than the huge rise in effective rms emittance for $\beta^{*}=1 \mathrm{~m}$. This clear benefit from a small mismatch is verified in Fig. 6. Further, the modified beta and alpha functions, calculated from the phase space distribution,

$$
\tilde{\beta}(s=0)=0.13 \mathrm{~m} \quad \tilde{\alpha}(s=0)=0.42,
$$

are closer to the design values than those at the case of $\beta^{*}=1 \mathrm{~m}$.

The nonlinear portion of the beam-beam force not only causes the growth of geometric emittance, but also leads to the formation of longer tails and a dense core in the electron beam distribution. The population of distribution tails creates a beam halo that may require larger acceptance of the accelerator elements to prevent beam losses during the deceleration of the electron beam. Simulation results, presented in Fig. 7, demonstrate the deformation of the electron beam distribution and the generation of the beam halo. In the simulations, we considered that the initial

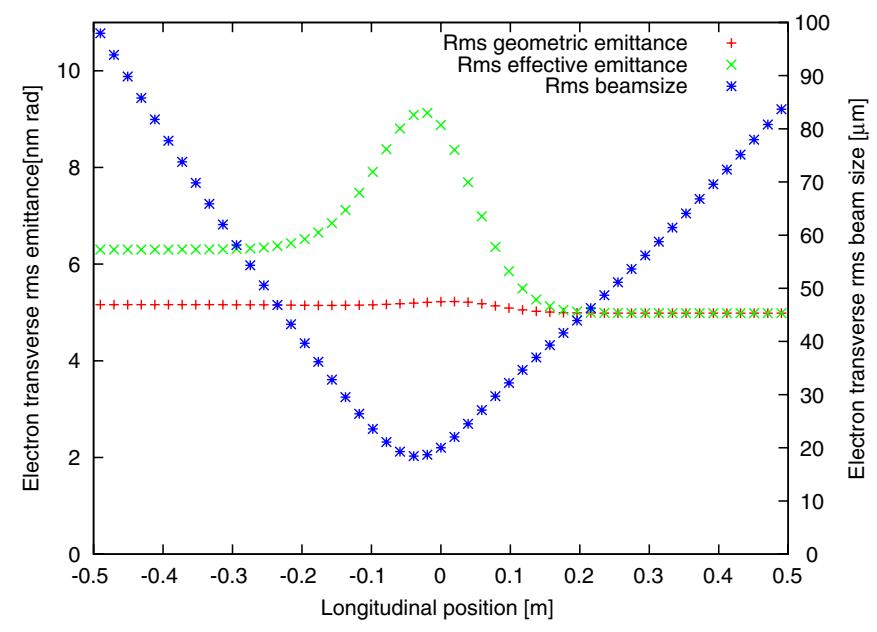

FIG. 5. (Color) Evolution of beam size and rms emittance (geometric and effective) of the electron beam for $\beta^{*}=0.2 \mathrm{~m}$ at $s^{*}=0 \mathrm{~m}$.

electron beam distribution, before the beam-beam collision, was Gaussian with a 4 sigma cutoff, so that all macroparticles are covered by the phase space ellipse with the area corresponding to 16 initial rms emittance. After the beam-beam interaction, the electrons populate betatron amplitudes to levels at least 25 times of the final rms emittance as shown in Fig. 7.

To evaluate the required acceptance of the accelerator elements, we considered $100 \%$ geometric and effective transverse emittance of electron beam, in addition to corresponding rms emittance values. For a Gaussian distribution it is apparent that the $100 \%$ emittance values directly depend on the convention for tail cutoff.

Figure 8 shows that there is still a large discrepancy between $100 \%$ geometric emittance and the effective emittance for the small initial emittance case $(1 \mathrm{~nm} \mathrm{rad})$. The discrepancy is becoming less if initial electron emittance is set to a larger value $(5 \mathrm{~nm} \mathrm{rad})$. The advantage of a large

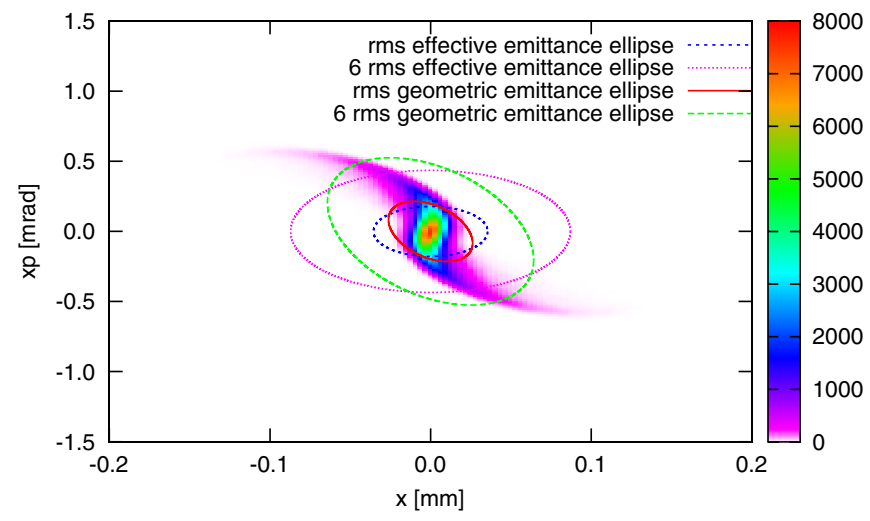

FIG. 6. (Color) Phase space distribution of the electron beam after collision for $\beta^{*}=0.2 \mathrm{~m}$, back traced to the IP. The rms and $6 \mathrm{rms}$ ellipses for both geometric and effective emittance, respectively, are plotted. 


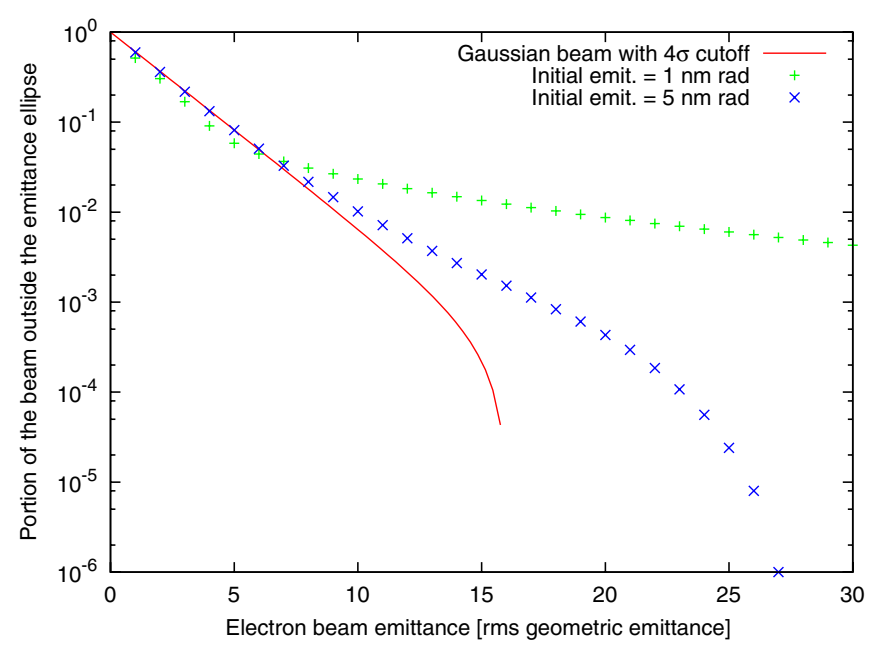

FIG. 7. (Color) The portion of electron beam after collision that is not covered by a given emittance ellipse as a function of the electron beam geometric acceptance (measured by the rms geometric emittance after the collision).

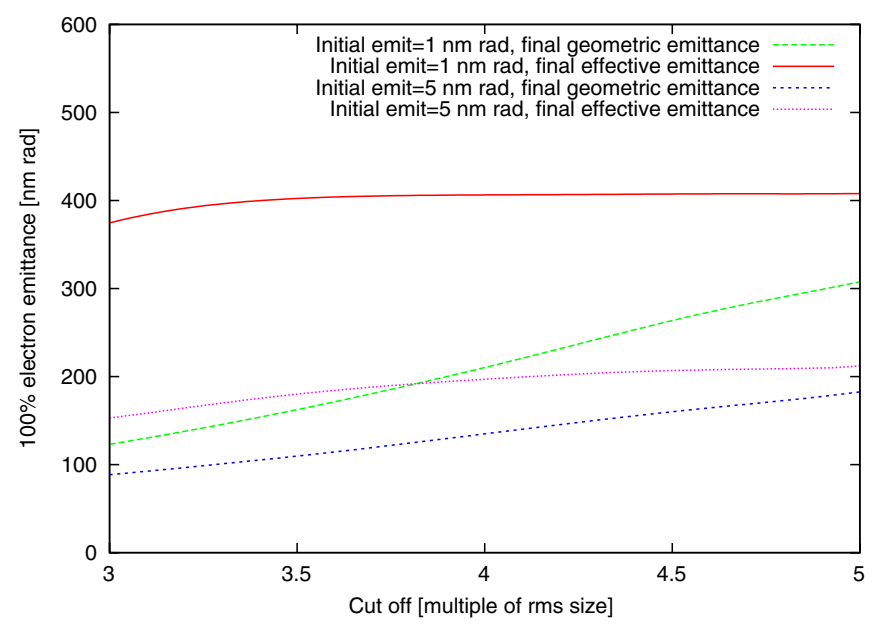

FIG. 8. (Color) $100 \%$ electron geometric and effective emittance as a function of cutoff points for Gaussian distribution, for a large (initial $\mathrm{rms}$ emittance $=5 \mathrm{~nm} \mathrm{rad} ; \beta^{*}=0.2 \mathrm{~m}$ ) and a small initial emittance (initial $\mathrm{rms}$ emittance $=1 \mathrm{~nm} \mathrm{rad} ; \beta^{*}=$ $1 \mathrm{~m})$.

initial electron emittance is still obvious; it yields higher beam quality after beam-beam collisions.

\section{LUMINOSITY ENHANCEMENT}

The luminosity numbers shown in the Table I are calculated using design values of beta functions and emittances, without the beam-beam interaction. However, under the strong focusing generated by the beam-beam force, the electron beam shrinks more extensively in the transverse directions than predicted from the design optics, as shown in Figs. 3 and 5 for different variants of the design optics. Because of this decline in size, called the pinch effect, the collision luminosity is enhanced.
To evaluate the extent of this luminosity enhancement, from the simulation data, the simulation code performs numerical integration, using the luminosity formula for a Gaussian beam distribution:

$$
L=\int \frac{2 N_{p} \lambda(2 s) N_{e} f_{c}}{2 \pi\left[\sigma_{p}^{2}(s)+\sigma_{e}^{2}(s)\right]} d s .
$$

Here, the parameters of the proton and electron beams are distinguished, respectively, by subscripts $p$ and $e . f_{c}$ is the collision frequency, $2 \lambda(2 s)$ is the normalized longitudinal distribution of the proton beam, and $\sigma_{e}(s)$ is determined from the distribution of macroparticles, including the evolution of this distribution throughout the collision area. Although, as we demonstrated in Sec. IV, the transverse distribution of the electron beam deviates from the Gaussian form because of the beam-beam interaction, Eq. (18) should supply a reasonably good approximation for the luminosity in the studied range of the eRHIC's beam parameters.

Since the electron beam disruption strongly depends on the choice of the design optics, so does the luminosity. Figure 9 and Table II present the evolution of the electron beam size throughout the IR and its corresponding luminosity, calculated using Eq. (18) for several variants of IR optics of the electron beam. In all cases illustrated, we selected the initial rms emittances of electrons to match the designed rms size of the proton beam at $s^{*}=0 \mathrm{~m}$ (without beam-beam interaction). The luminosity is lower for smaller design $\beta^{*}$ values, partly because there is less pinching of the beam, and partly due to the "hourglass" effect.

Unfortunately, for eRHIC, the enhancement in luminosity cannot be used effectively because pinching of the electron beam increases the beam-beam force acting on the proton beam. With the proton beam-beam parameter as

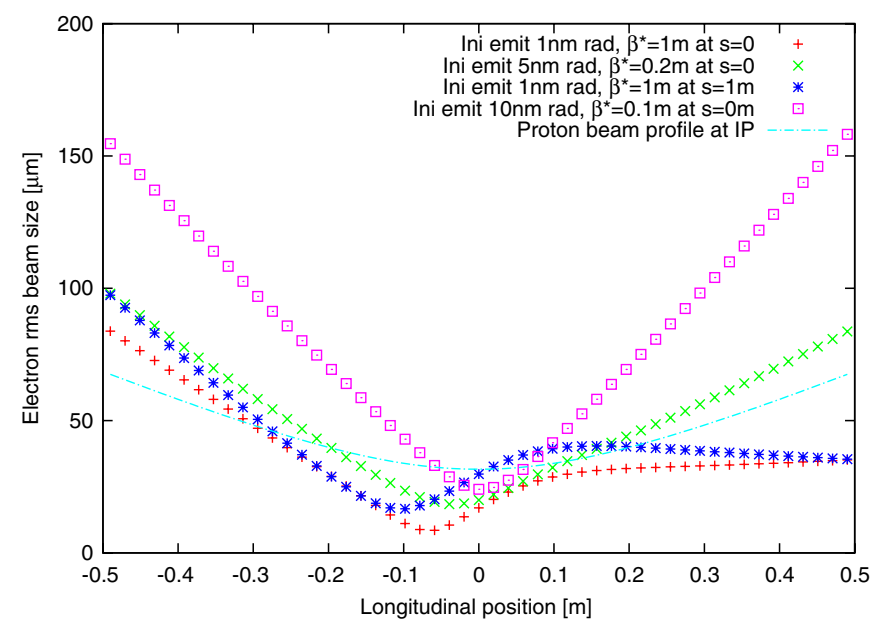

FIG. 9. (Color) Evolution of the size of the electron and proton beam under different optics and initial emittances. The proton beam travels from left to right, and the electron beam travels from right to left. 
TABLE II. Luminosity at different sets of initial electron beam parameters.

\begin{tabular}{lccc}
\hline \hline Emittance [nm rad] & $\beta^{*}[\mathrm{~m}]$ & $s^{*}[\mathrm{~m}]$ & Luminosity $\left[\mathrm{cm}^{-2} \mathrm{~s}^{-1}\right]$ \\
\hline 1 & 1 & 0 & $3.4 \times 10^{33}$ \\
1 & 1 & 1 & $2.6 \times 10^{33}$ \\
4 & 0.25 & 0 & $3.0 \times 10^{33}$ \\
5 & 0.2 & 0 & $2.9 \times 10^{33}$ \\
10 & 0.1 & 0 & $2.1 \times 10^{33}$ \\
\hline \hline
\end{tabular}

large as 0.015 , further increase in the beam-beam force may result in the slow growth of its emittance, as demonstrated in the simulation results. In addition, strong pinching correlates with strong mismatch of electron beam relative to the design optics, along with larger growth of the geometric emittance, as discussed in previous sections.

We accounted for the modification of the luminosity by beam-beam interactions, during our studies of the optics optimization studies described in Sec. VI.

\section{ELECTRON BEAM OPTICS OPTIMIZATION}

As we discussed earlier, the deterioration of the electron beam's geometric and effective emittances due to processes of the disruption and mismatch caused by beambeam collisions is a strong function of design optics and initial emittance of the electron beam. We observed a general trend towards improvements in both emittances after the collision when the electron design beta function was lowered (from $\beta^{*}=1 \mathrm{~m}$ to $\beta^{*}=0.2 \mathrm{~m}$ ) and the initial emittance of electrons increased (from 1 to $5 \mathrm{~nm} \mathrm{rad}$ ) to maintain the match between the designed electron beam size at the IP and the proton beam size. The collision luminosity is a function of the design optics (including the hourglass effect). It depends also on the modifications of the electron beam transverse size throughout the IR engendered by the beam-beam interaction. Accordingly, a question can be asked about the optimal choice of electron lattice functions and the initial transverse emittance that would provide high luminosity at minimized electron beam disruption. To find those optimized parameters, we undertook detailed simulations.

During the simulations, the following parameters of electron beam were varied: the initial transverse emittance (before the collision), the design beta function at the waist $\left(\beta^{*}\right)$, and the longitudinal position of the beta-function waist $\left(s^{*}\right)$. The first two determine the minimum of rms electron beam size without the collisions; the third also presents an important parameter. Intuitively, with a large enough positive $s^{*}$, the divergence of electron beam at the entrance of IR should aid in reducing the pinch effect, so that approximately constant electron beam size is achieved throughout the collision zone.

During these optics optimization studies, we abandoned the requirement of matching the design transverse sizes of the electron and proton beam at the IP to perform a full investigation. Thus, the variations of all three electron beam parameters were not correlated in any way so that the 3D parametric space was covered with the parameter ranges shown in Table III. The main parameters of interest at the simulation output are the luminosity and the geometric and effective emittances. For the emittances we looked at the output for both the rms emittances and $100 \%$ emittances, thereby to follow the corresponding changes in the beam core and the beam halo. Since the initial transverse distribution of macroparticles representing the electron beam is Gaussian with 4 sigma cutoff, $100 \%$ emittance is a finite number.

The parameters of the proton beam were unchanged during these studies; they are listed in Table I. Thus the electron beam disruption parameter, defined by Eq. (7), remained the same for all presented data.

For a reasonable comparison, we grouped all the output as a function of luminosity. Figures 10-12 present the simulation results where the output data are shown as the dependencies on luminosity of various beam emittances after collision. Different symbols correspond to different initial electron beam emittances. Each set of points for an individual initial emittance includes the points corresponding to various design $\beta^{*}$ and $s^{*}$ from Table III.

Although we give a two-parametric set of points for each individual emittance, all figures show remarkable correlation between the final emittances and the luminosity. The differences in optics, for a given initial emittance, which lead to larger disruption and larger mismatch, also produce smaller luminosity.

Figure 10 shows the geometric rms emittance after the collision versus the luminosity. Large luminosity corresponds to smaller average beam size, and expectedly, smaller beam disruption by the nonlinear part of the beam-beam field. A remarkable feature is that at any given luminosity the final rms emittance depends only weakly on the initial emittance value. While the initial emittances range from 1 to $8 \mathrm{~nm}$, the geometric rms emittances after the collision are within $50 \%$, at given luminosity value.

TABLE III. Table of example parameters used in the optics optimization (design values are marked with \#).

\begin{tabular}{lc}
\hline \hline Initial electron emittance $\left(\times 10^{-9} \mathrm{~m} \mathrm{rad}\right)$ & $1 \#, 2,3,4,5,6,7,8$ \\
\hline$\beta^{*}(\mathrm{~m})$ & $0.1,0.2,0.4,0.6,0.8,1 \#, 1.5,2$ \\
$s^{*}(\mathrm{~m})$ & $-0.5,-0.4,-0.3,-0.2,-0.1,-0.05,0 \#, 0.1,0.2$ \\
\hline \hline
\end{tabular}




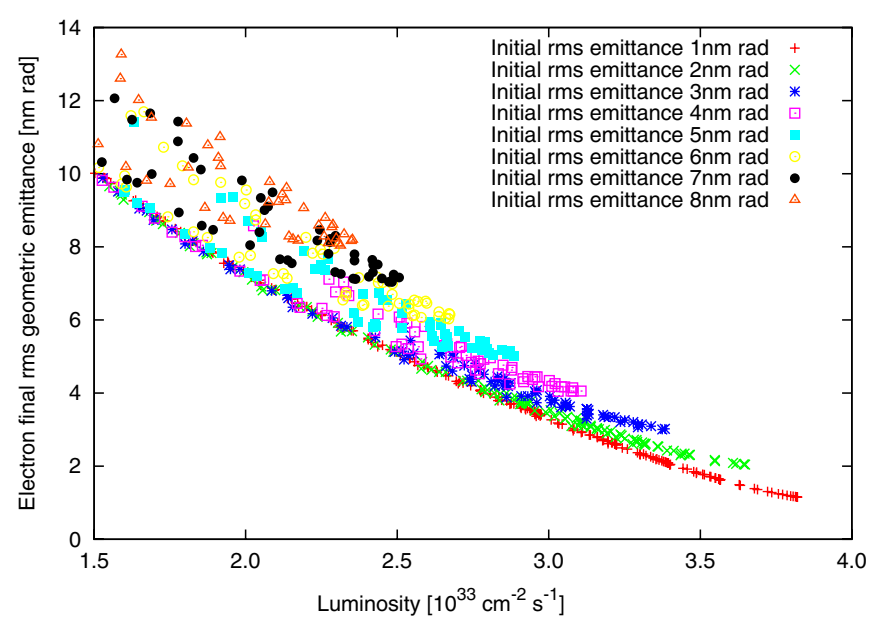

FIG. 10. (Color) Electron beam rms geometric emittance after collision as a function of luminosity.

On the contrary, the rms effective emittance of the electron beam after collision based on the design lattice strongly depends on the initial emittance (as seen in Fig. 11). Thus, for a fixed target luminosity, there must be a larger initial emittance to achieve smaller final rms effective emittance.

Figure 12 demonstrated that, for $100 \%$ effective electron emittance after collision, the dependence on the luminosity is similar to that of rms effective emittance. For any given luminosity, a larger initial emittance is preferable in achieving smaller final $100 \%$ emittance. For the initial rms emittances greater than $4 \mathrm{~nm} \mathrm{rad}$, the improvements in reduction of effective emittances become small.

All plots show that smaller final emittances are attained at higher luminosity. However, another factor limits the realizable luminosity which is the effect on the proton beam. Figure 13 plots the correlation between the luminosity and the average electron beam size. The smaller electron beam size enhances the beam-beam force acting

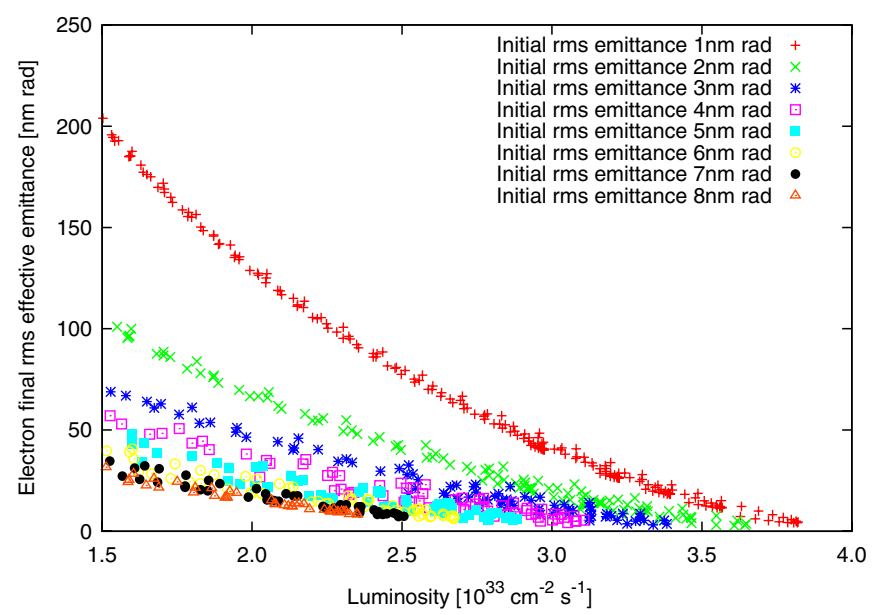

FIG. 11. (Color) Electron beam rms effective emittance after collision as a function of luminosity.

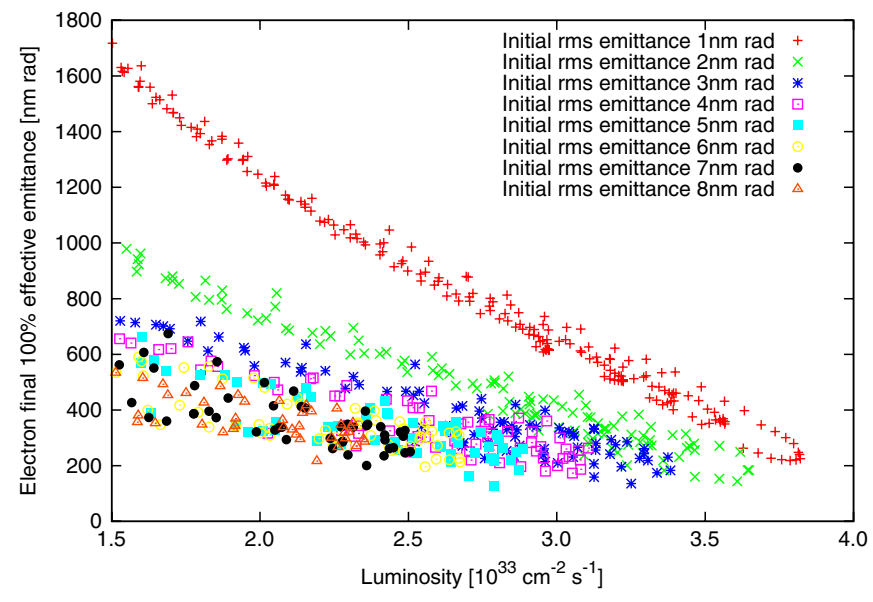

FIG. 12. (Color) Electron beam 100\% effective emittance after collision as a function of luminosity.

on the protons and leads to the slow deterioration of the proton beam emittance. We will present detailed results for this effect on the proton beam in eRHIC in another paper. They put the limit on the achievable luminosity approximately at the $2.8 \times 10^{33} \mathrm{~cm}^{-1} \mathrm{~s}^{-1}$ level.

Summarizing the results of the electron beam parameter scan, we conclude that, to minimize the final electron beam emittance after the collision, its initial emittance should not be less than $4 \mathrm{~nm}$ rad. Thus, the initial design emittance value of $1 \mathrm{~nm}$ rad and the original design of the IR must be reconsidered to minimize the electron beam disruption and mismatch. All evidence suggests we select a larger initial emittance and corresponding small $\beta^{*}$. However, the hourglass effect prevents us from choosing initial emittance larger than $6 \mathrm{~nm}$ rad because of the reduction on the luminosity, as illustrated in Figs. 10-12. The ideal initial emittance should be 4 to $5 \mathrm{~nm}$ rad to achieve both acceptable electron beam quality after collision and large luminosity. To perform further beam loss study, our new

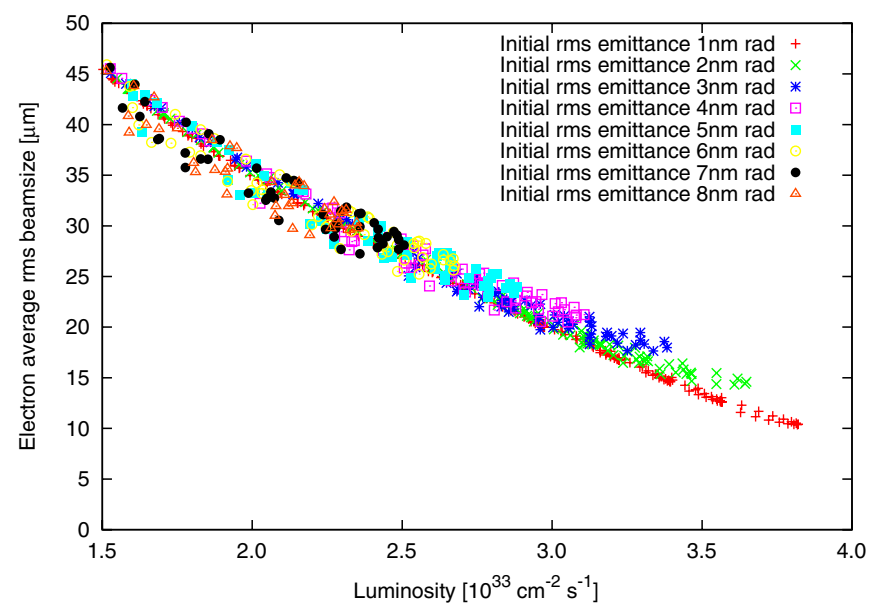

FIG. 13. (Color) Average electron beam size in IR as a function of luminosity. 


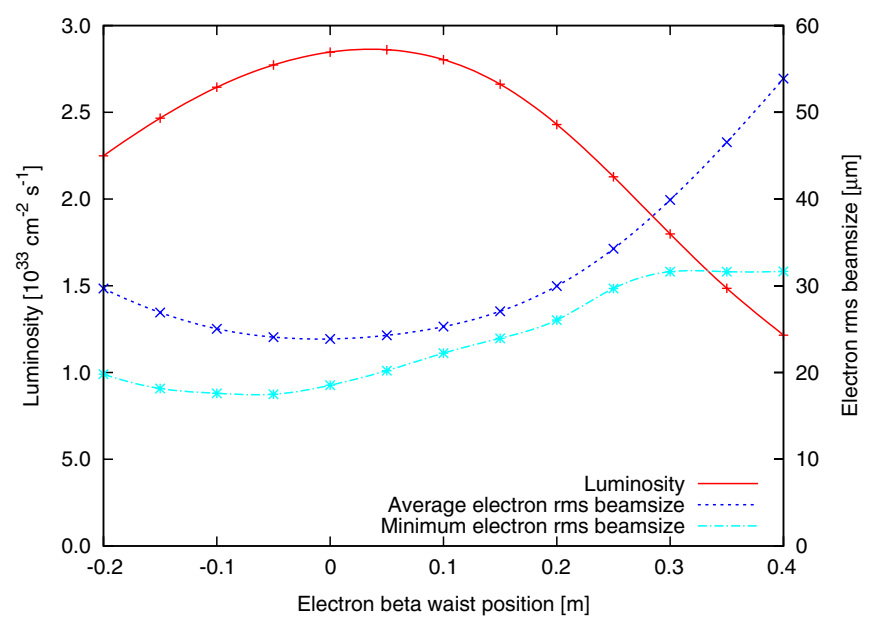

FIG. 14. (Color) Dependence of luminosity and the electron beam size as a function of $s^{*}$.

choice of electron beam parameters is set as emittance $=$ $5 \mathrm{~nm} \mathrm{rad}$ and $\beta^{*}=0.2 \mathrm{~m}$. For this parameter choice, with the waist position $s^{*}$ as the left free parameter, we illustrate in Fig. 14 luminosity and the electron beam size as a function of $s^{*}$. The maximum luminosity and lowest average electron beam size are achieved simultaneously with the beta waist close to the IP $\left(s^{*}=0 \mathrm{~m}\right)$. However, considered the pinch effect, we can select the parameter $s^{*}$ with a positive value as a compromise between the high luminosity and proton beam stability.

Similar beam parameter optimization was done for lower energy collision setup, $3 \mathrm{GeV}$ electrons on $50 \mathrm{GeV}$ protons. Optimized electron beam parameters are shown in Table III in parentheses.

\section{BEAM LOSS ESTIMATION AND MATCHING SCHEME}

The eRHIC scheme exploits the principle of the energy recovery so that, after passing the collision point, the electron beam must be transported again through the long recirculation passes and decelerated in the linac. From our results on disruption effect of the electron beam, we can assess the required aperture of the recirculation-pass magnets that will accommodate the transport of the electron beam after its collisions. The question about the size of magnet aperture is important because using compact magnets significantly reduces the machine's overall cost.

In the following evaluation we assumed that, after the collision, the beam is transported through the linear optics of the recirculation passes, wherein the maximum $\beta$ function is about 50 meters. The initial electron rms emittance (before the collision) is $5 \mathrm{~nm}$ rad and $\beta^{*}$ is 0.2 meters. The beam-beam interaction leads to the geometrical emittance after the collisions about $5.2 \mathrm{~nm}$, and the effective emittance around $6.3 \mathrm{~nm}$.

The beam loss occurs when large-amplitude electrons exceed the aperture of the recirculation pass or linac. As the beam decelerates and its transverse size grows adiabatically, the number of electrons lost for a given magnet aperture increases. However, the evaluation of beam loss power calls for more careful consideration because the energy of the lost particles declines with the beam's energy. The beam power loss for a given aperture $a$ is

$$
P=2 \pi m c^{2} \gamma f \int_{J_{a}=\gamma a^{2} / 2 \beta}^{\infty} \rho(J) d J,
$$

where $J$ is the normalized action and $J_{a}$ is the normalized action aperture. For simplicity, we assumed that the distribution function $\rho$ depends only on action. From here, the calculated energy dependence of the power loss yields

$$
\frac{d P}{d \gamma}=2 \pi m c^{2} f\left(\int_{J_{a}}^{\infty} \rho(J) d J-J_{a} \rho\left(J_{a}\right)\right) .
$$

Taking Gaussian distribution $\rho(J) \propto \exp \left(J / J_{0}\right)$ as an instance, we find that

$$
\frac{d P}{d \gamma}=2 \pi m c^{2} f\left(J_{0}-J_{a}\right) \rho\left(J_{a}\right)
$$

$d P / d \gamma<0$ when $J_{a}>J_{0}$ which is always true because the magnet aperture is always much larger than the rms beam size. This implies the power of the beam losses inevitably increases as the electron beam decelerates. Therefore the special attention should be focused on lower energy passes.

We present our results for two different cases. In the first, the electron beam's collision energy is $10 \mathrm{GeV}$, and its lowest energy of the recirculation pass is $2.4 \mathrm{GeV}$. In the second, the electron beams collide at $3 \mathrm{GeV}$ and the corresponding lowest energy in the recirculation pass in $1.75 \mathrm{GeV}$. For both cases, our study of beam loss power also looks at beam energies of $0.5 \mathrm{GeV}$ (the energy in the transport line connecting the main and preaccelerator linac) and $10 \mathrm{MeV}$ (the energy in the transport line to the beam dump). All of the results presented below refer to the aperture as to the radius of the beam pipe in the magnets.

Figure 15 plots the beam power losses versus aperture for $10 \mathrm{GeV}$ collision energy and two possible initial transverse distributions, i.e., Beer-Can and Gaussian. As earlier in this paper the initial Gaussian distribution is cut at 4 sigma. The Beer-Can distribution entails smaller aperture requirements since less beam halo is created by the collision. According to Fig. 15, the aperture of lowest energy recirculation-pass magnets $(2.4 \mathrm{GeV})$ should be at least $7 \mathrm{~mm}$ for Beer-Can distribution, and at least $16 \mathrm{~mm}$ for Gaussian. The real distribution of the electron beam is expected to be somewhere between the Beer-Can and Gaussian distribution, thus, the least magnet aperture should reside between those two numbers.

Our analysis of the low collision energy ( $3 \mathrm{GeV}$ electron beam) follows the same steps as that for the $10 \mathrm{GeV}$ energy case. The disruption parameters for both energies are close to each other. Figure 16 depicts the similar features of the power loss for the low-energy and the high-energy case. 


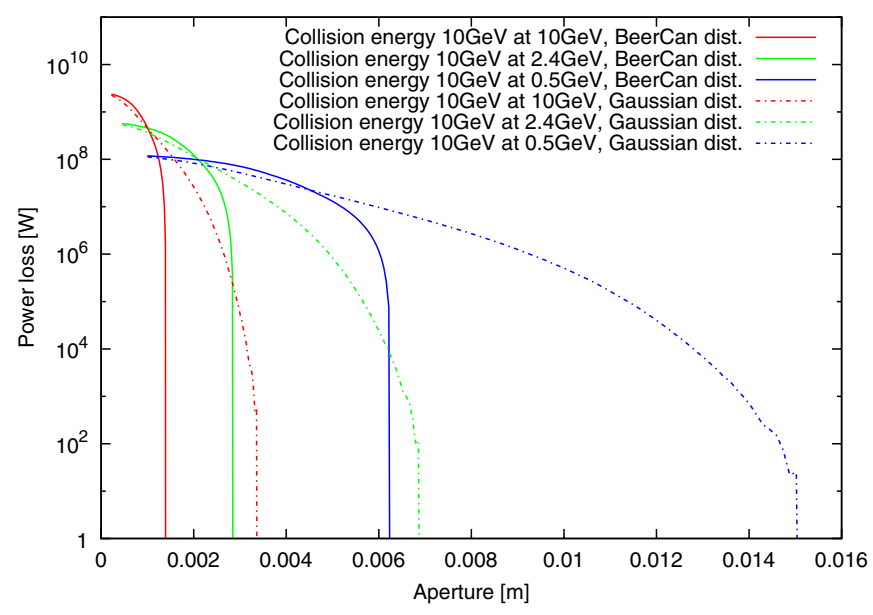

FIG. 15. (Color) Power loss at a given aperture and energy with the initial Gaussian distribution and Beer-Can distribution, highenergy setup (10 GeV collision energy).

As the comparison in Fig. 17 shows, a larger aperture is needed to avoid the beam losses after the low-energy collision than after the high-energy one. That is due to a more pronounced effect of the nonlinear beam-beam force on a less rigid beam. However, the difference in the aperture requirements for different collision energies is small. For both energy cases, the estimated apertures are around $16 \mathrm{~mm}$ when the beam is decelerated to $0.5 \mathrm{GeV}$.

As the aperture requirement increases at lower beam energies, we might consider applying a special matching scheme in the IR to compensate for the mismatch effect caused by the beam-beam interactions. With such a scheme, the IR optics downstream of the collision point is adjusted to assure a perfect match of lattice functions ( $\beta$ and $\alpha$ functions), distorted by the collisions, with the optics of the following recirculation pass. Then, the geometric emittance rather than the effective one better characterizes

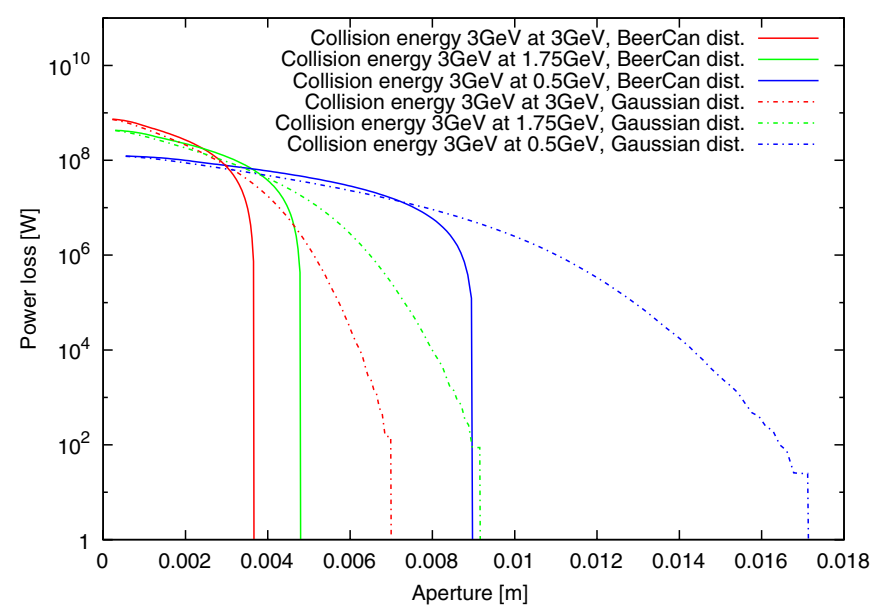

FIG. 16. (Color) Power loss at a given aperture and energy with the initial Gaussian distribution and Beer-Can distribution, lowenergy setup ( $3 \mathrm{GeV}$ collision energy).

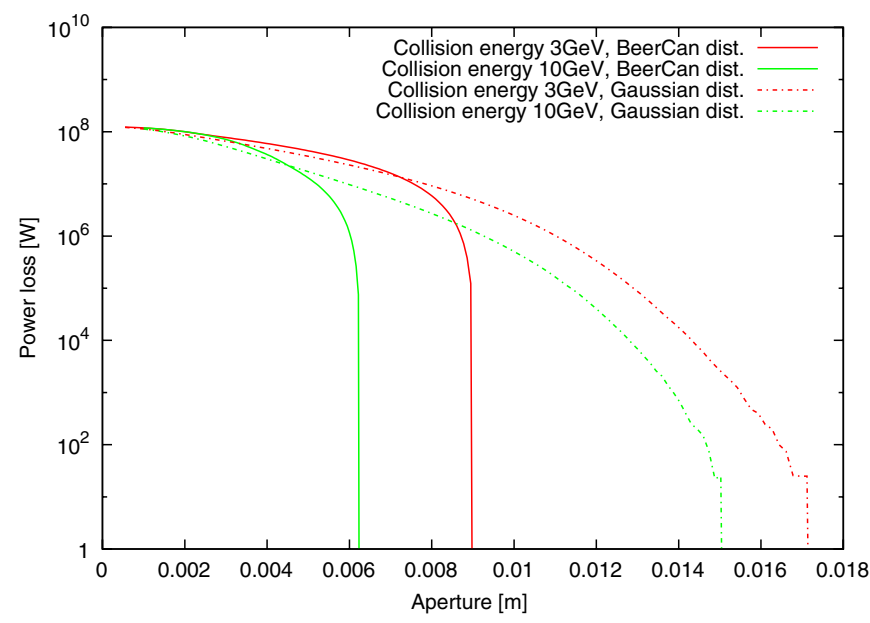

FIG. 17. (Color) Comparison of power loss at $0.5 \mathrm{GeV}$, between different collision energies of the electron beam, 10 and $3 \mathrm{GeV}$.

the increase in the beam's emittance. As the former is usually smaller than the latter, we anticipate a corresponding improvement in the aperture's requirements. However, to obtain a good assessment of beam loss power and
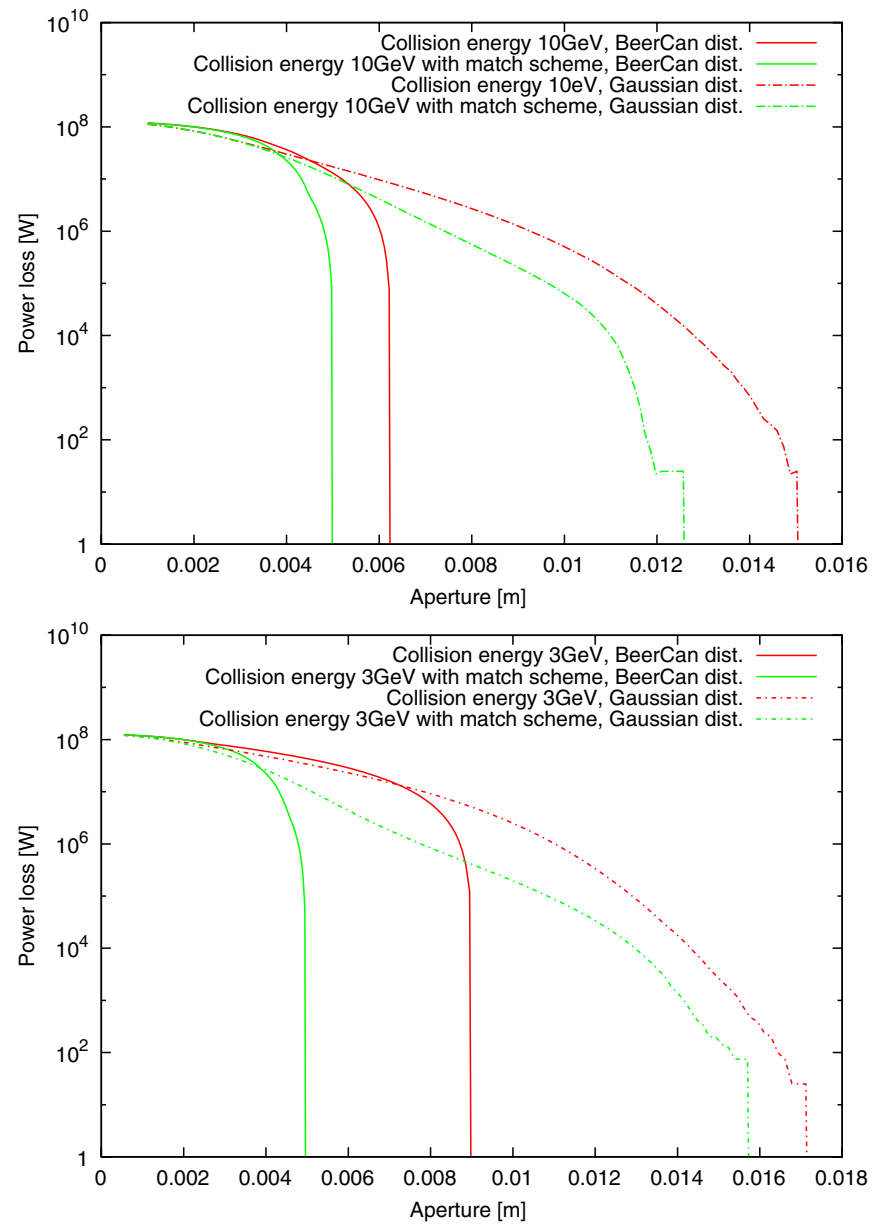

FIG. 18. (Color) Power loss at $0.5 \mathrm{GeV}$ with or without matching scheme; in the top graph the collision energy is $10 \mathrm{GeV}$ and in the bottom graph it is $3 \mathrm{GeV}$. 


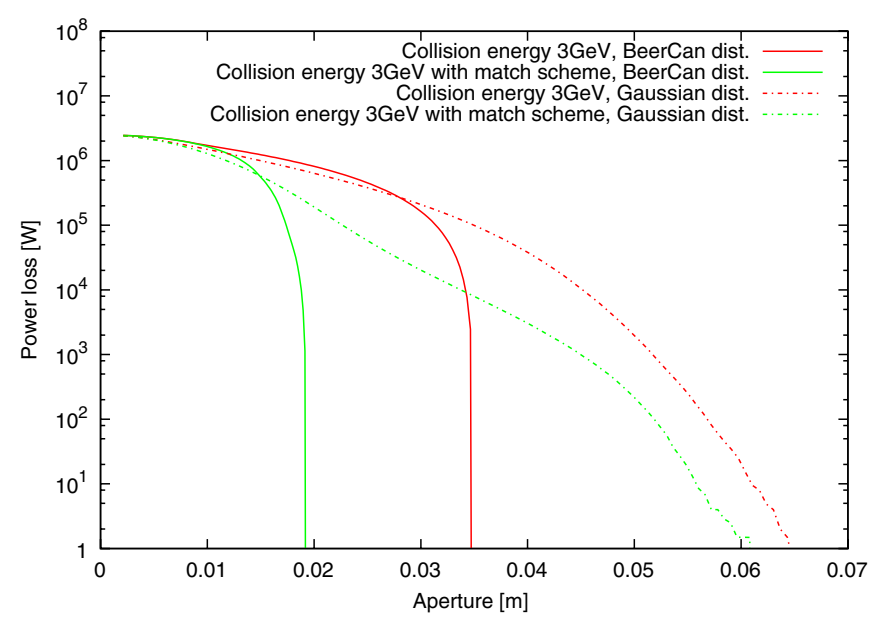

FIG. 19. (Color) Power loss at $10 \mathrm{MeV}$ with or without matching scheme for low collision energy setup ( $3 \mathrm{GeV})$.

aperture requirements, it is important to consider the tails of electron distribution.

Figure 18 illustrates that, for both the high collision energy setup and the low collision energy setup, applying the matching scheme effectively decreases the beam loss power only for the Beer-Can distribution. In contrast, for a Gaussian beam, the matching scheme loses its effectiveness, because the halo of electron distribution, formed by beam-beam force nonlinearity, is insensitive to this linear compensation. Fortunately, it takes the damping time, in order of milliseconds, to form a Gaussian shape distribution, which is much longer than the transport time from the source to IP in ERL. Figure 19 shows the results for the least required aperture in the $10 \mathrm{MeV}$ transport line to the beam dump. Applying a matching scheme lowers the least aperture required by an initial Beer-Can distribution by a factor of $40 \%$.

We conclude that, provided the electron distribution before the collision does not have long tails, as in a Gaussian distribution, the matching scheme will reduce the required aperture by about $40 \%$. Should this reduction be deemed essential for the magnet design of the energyrecovery passes, it would be worthwhile to incorporate the matching scheme into the interaction region design.

In the case of Gaussian distribution the collimation may be needed at some stage during the beam deceleration in order to provide better protection of superconducting $\mathrm{rf}$ cavities of the energy-recovery linac during the deceleration to $10 \mathrm{MeV}$.

\section{CONCLUSION}

We thoroughly explored the features of electron beam disruption in an ERL-based eRHIC. The electron beam geometrical emittance increases on colliding with an opposing proton beam. Also, the additional phase advance caused by the beam-beam interaction induces a mismatch between the electron beam transverse distribution and the design optics, and increases effectively the beam emittance.

We optimize the interaction region lattice parameters and the initial electron beam emittance, and demonstrate the advantages of a smaller waist beta function $\left(\beta^{*}\right)$ and a larger initial emittance for counteracting the emittance growth due to the beam-beam field nonlinearity and the mismatch. Table I shows in parentheses a new set of suggested electron beam parameters.

The electron beam halo created by the beam-beam interaction plays an important role in determining a required magnet aperture to avoid the beam losses when the electrons are decelerated on the energy-recovery path. We perform complete evaluation of the least required magnet aperture for different collision energies of electrons, different energies during the deceleration as well as different initial transverse distributions. If the optimization of the design cost will call for magnets with even smaller apertures, a dedicated matching scheme can be incorporated into the interaction region to provide up to $40 \%$ aperture reduction.

\section{ACKNOWLEDGMENTS}

We would like to thank Vladimir Litvinenko, Eduard Pozdeyev, and Yun Luo for their help in this work and constructive suggestions. This work is supported by the U.S. Department of Energy under Contract No. DE-AC0298CH10886.

[1] V. Ptitsyn et al., eRHIC Accelerator Position Paper, Technical Report (C-AD, BNL, 2007).

[2] V. Ptitsyn et al., eRHIC Zeroth-Order Design Report, Technical Report (C-AD, BNL, 2004).

[3] E. Keil, in CERN Report No. 95-06, 1994.

[4] S. A. Heifets, G. A. Krafft, and M. Fripp, Nucl. Instrum. Methods Phys. Res., Sect. A 295, 286 (1990).

[5] Y. Hao, Ph.D. thesis, Indiana University, 2008.

[6] S. Y. Lee, Accelerator Physics (World Scientific, Singapore, 1999). 